\title{
Molecular cloning and functional expression of human cytosolic acetyl-CoA hydrolase
}

\author{
Naoya Suematsu and Fumihide Isohashi ${ }^{\bowtie}$ \\ Department of Biochemistry, St. Marianna University School of Medicine, Kanagawa, Japan; \\ 凶e-mail: n2sue@marianna-u.ac.jp
}

Received: 09 March, 2006; revised: 09 June, 2006; accepted: 27 June, 2006

available on-line: 02 September, 2006

\begin{abstract}
A cDNA encoding human cytosolic acetyl-CoA hydrolase (CACH) was isolated from a human liver cDNA library, sequenced and functionally expressed in insect cells. The human CACH cDNA encodes a 555 -amino-acid sequence that is $81.4 \% / 78.7 \%$ identical to those of the mouse/rat homologue, suggesting a conserved role for this enzyme in the human and rodent livers. Bioinformatical study further reveals a high degree of similarity among the human and rodent CACHs as follows: First, the gene is composed of 15 exons ranging in size from 56 to $157 \mathrm{bp}$. Second, the protein consists of two thioesterase regions and a C-terminal steroidogenic acute regulatory protein-related lipid transfer (START) domain. Third, the promoter region is GC-rich and contains GC boxes, but lacks both TATA and CCAAT boxes, the typical criteria of housekeeping genes. A consensus peroxisome proliferator responsive element (PPRE) present in the rodent CACH promoter regions supports marked CACH induction in rat liver by peroxisome proliferator (PP).
\end{abstract}

Keywords: acetyl-CoA hydrolase, PCR, cDNA sequence, Spodoptera frugiperda, functional expression, housekeeping-type promoter

\section{INTRODUCTION}

The cytosolic or extramitochondrial acetylCoA hydrolase $(\mathrm{CACH})$ hydrolyzes acetyl-CoA to acetate and CoA. It seems superficially wasteful to hydrolyze the most common energy-rich metabolite acetyl-CoA without recovering any energy. So it is exciting to understand the physiological role of the enzyme comprehensibly.

The enzyme has been detected in rat liver (Prass et al., 1980) and kidney (its cytosolic CACH specific activity was $5 \%$ of that of the liver enzyme) (Matsunaga et al., 1985). Its activity in the liver increases notably in the opposite metabolic states: during enhanced fatty acid oxidation and in increased fatty acid synthesis (Matsunaga et al., 1985). Further, a marked induction in the liver was observed by thyroid hormones (Matsunaga et al., 1985) and also by 2-( $p$-chlorophenoxy) isobutyric acid (Ebisuno et al., 1988), a hypolipidemic drug or peroxisome proliferator $(\mathrm{PP})$, which enhances $\beta$-oxidation in rat liver mitochondria and peroxisomes (Mannaerts et al., 1979) and increases cytosolic CoA level (Berge et al., 1983; Horie et al., 1986). These findings reveal that the enzyme plays a vital role specifically in fat metabolism by supplying cytosolic free CoA necessary for both fatty acid synthesis and oxidation (Matsunaga et al., 1985).

The enzyme had rejected earlier an enough of purification due to its extreme cold lability (Isohashi et al., 1983a; Suematsu et al., 1996) and little activity in the absence of ATP (Söling \& Rescher,

^Note: Nucleotide sequence data are available in the DDBJ/EMBL/GenBank databases under the accession number AB078619. Enzymes: acetyl-CoA hydrolase (EC 3.1.2.1); acyl-CoA thioesterase (EC 3.1.2.2); 4-hydroxybenzoyl-CoA thioesterase (EC 3.1.2.23).

Abbreviations: $\mathrm{CACH}$, cytosolic acetyl-CoA hydrolase; ESTs, expressed sequence tags; NCBI, National Center for Biotechnology Information; TESS, Transcription Element Search Software; ORF, open reading frame; START, steroidogenic acute regulatory protein-related lipid transfer; 4HBT, 4-hydroxybenzoyl-CoA thioesterase; PP, peroxisome proliferator; PPRE, peroxisome proliferator responsive element; bHLH, basic helix-loop-helix. 
1985; Nakanishi et al., 1988). At long last, we successfully purified the enzyme in the presence of a protease inhibitor at room temperature (Ebisuno et al., 1989; Nakanishi et al., 1993). Characterization of the purified enzyme demonstrated that at room temperature it was usually present as active forms: homodimer (135 kDa) and homotetramer $(240 \mathrm{kDa})$ whose $K_{\mathrm{m}}$ values for acetyl-CoA were $170 \mu \mathrm{M}$ and $60 \mu \mathrm{M}$, respectively (Isohashi et al., 1983a). But under cold conditions, they dissociate into an inactive monomer $(63 \mathrm{kDa})$ showing no hydrolyzing activity any more even at $25^{\circ} \mathrm{C}$ (Isohashi et al., 1984). Our in vitro study further revealed that $\mathrm{CACH}$ is an allosteric enzyme regulated by ATP (activator) and ADP (inhibitor) (Isohashi et al., 1983b; Nakanishi et al., 1994), suggesting it is presumably a key enzyme involved in energy metabolism. It should be noted here that ATP is not a substrate but an allosteric effector because ATP works well without production of either ADP or inorganic phosphate in the absence of $\mathrm{Mg}^{2+}$ (Prass et al., 1980). Recently, we found that low concentration of Triton X-100 prevents cold inactivation of $\mathrm{CACH}$ and further partially reactivates the cold-inactivated enzyme at $37^{\circ} \mathrm{C}$ (Suematsu et al., 2003), allowing us to purify the enzyme efficiently under usual cold conditions at $4^{\circ} \mathrm{C}$.

We previously reported molecular cloning of rat and mouse $\mathrm{CACH}$ CDNAs, demonstrating that the enzyme is a novel thioesterase (Suematsu et al., 2001; 2002). Here we report molecular cloning and recombinant expression of a human homologue cDNA, as the third example of mammalian species. We have further analyzed the corresponding gene in the established databases and describe its exonintron structure. We also present an initial search for its cis-regulatory elements. Molecular analysis of the newly discovered human $\mathrm{CACH}$ gene might provide clues to its expression and physiological functions of the enzyme and further our understanding of the implications of peroxisome proliferator-induced pleiotropic responses to human health.

Table 1. PCR primers used for cloning human acetyl-CoA hydrolase cDNA

Nucleotide positions are numbered as in Fig. 2. The restriction site used for the cDNA cloning is highlighted in bold type. S, sense; A, antisense; CDS, coding sequence.

\begin{tabular}{ll}
\hline Primer & Sequence $\left(5^{\prime}-3^{\prime}\right)$ \\
\hline S0 & AAATTTGAACGCCAGCACATGGAC \\
S1 & GCCGCAGGCTTAGCGCTCTCG \\
S2 & GGCTTAGCGCTCTCGCCCTGG \\
S3 & TTCAGAGCATTGAACTGGTCCTCC \\
S4 & AAAAGGGGTTGGGAGGTTACCAGC \\
S5 & TCTCGCCCGGGCCATGGAGC \\
A0 & TCGATCTCAGTGGTATTTGTGAGC \\
A1 & CCAAATCACTGGTAGACAAGATGC \\
A2 & CTCCGTCACTGCATTTTGCTTAGG \\
A3 & TTTTCCACAGTGCTGGTAACCTCC \\
A4 & AAATGTGTTTCCGTGGTGGTTTGC
\end{tabular}

Position

Location

\begin{tabular}{lc}
\multicolumn{2}{c}{ (specific to pTriEx-4 vector) } \\
$-29 /-9$ & $5^{\prime}-$ UTR \\
$-23 /-3$ & $5^{\prime}$-UTR \\
$548 / 571$ & CDS \\
$1093 / 1116$ & CDS \\
$-13 / 7$ & anchor primer \\
\multicolumn{1}{l}{ (specific to pTriEx-4 vector) } \\
$1809 / 1832$ & $3^{\prime}$-UTR \\
$1774 / 1797$ & $3^{\prime}$-UTR \\
$1104 / 1127$ & CDS \\
$580 / 603$ & CDS
\end{tabular}


down approach. The resulting recombinant transfer vector was designated as pTriEx-4/hCACH (Fig. 1A).

Recombinant expression and affinity purification. The procedures for amplification of the recombinant virus and the assessment of recombinant expression were previously described in detail (Suematsu et al., 2002). The recombinant virus was isolated and then used to transform monolayer Spodoptera frugiperda (Sfg) insect cells at a multiplicity of infection of 10 for recombinant expression of the His-tag fusion protein (Fig. 1B). Affinity purification of the expressed human His-tag $\mathrm{CACH}$ was performed on $\mathrm{Ni}^{2+}$-charged resin using HISTAGcatcher $(\mathrm{Cy}-$ tosignal) according to the procedures provided with the purification system. The purified His-tag fusion protein was then relieved of the fused peptide by thrombin cleavage using Thrombin Cleavage Capture Kit (Novagen) according to the manufacturer's instructions.

Bioinformatics. The nucleotide sequences of the non-coding region of mammalian $\mathrm{CACH}$ genes were from established databases. Nucleotide and amino-acid sequence homology was searched with a computer using BLAST 2.0, and sequence alignments were made using the DiAlign computer algorithm (Genomatix Software $\mathrm{GmbH}$, München). Subcellular localization was predicted by PSORT WWW Server (http://psort.nibb.ac.jp). Functional domains were predicted using the National Center for Biotechnology Information (NCBI) Conserved Domain Search website (www.ncbi.nlm.nih.gov/Structure/cdd/ wrpsb.cgi). Consensus cis-elements were searched using the Transcription Element Search Software (TESS) website (Schug \& Overton, 1997) browsing the TRANSFAC database.

A

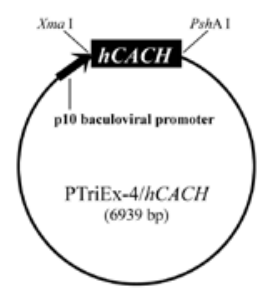

B

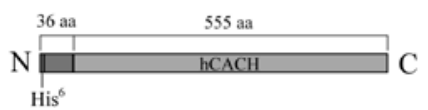

Figure 1. Schematic depiction of the strategy for recombinant expression of a full-length ORF of human cytosolic acetyl-CoA hydrolase.

A. Construction of the recombinant baculovirus transfer vector containing the complete coding region of human cytosolic acetyl-CoA hydrolase (hCACH). The full-length ORF encoding hCACH was inserted into the multiple cloning site of the baculovirus transfer vector pTriEx-4 at the XmaI/PshAI site. The resultant vector was $6939 \mathrm{bp}$ long and designated as pTriEx- $4 / h C A C H$. B. A schematic representation of the recombinant $\mathrm{CACH}$ containing a polyhistidine $\left(\mathrm{His}^{6}\right)$ tag.

\section{RESULTS}

Molecular cloning and sequence analysis of a human CACH cDNA covering a full-length ORF

Database searching revealed that several human expressed sequence tags (ESTs) represent high homology (around 75\%) to the rodent $\mathrm{CACH} \mathrm{cD}$ NAs (Suematsu et al., 2001; 2002), implicating the existence of a human homologue cDNA. One human EST clone (586 bp long, AV693695 in GenBank) shows $82 \%$ identity to the 5 '-terminus of the rat cDNA in a 114 amino acid overlap, and another (394 bp long, AV685167 in GenBank) shows 70\% identity to the 3 '-terminus in a 73 amino acid overlap. Based on the nucleotide sequences of the two ESTs, oligonucleotide PCR primers were designed (Table 1) and used to clone a human homologue cDNA from a human liver cDNA library. As outlined in Fig. 2, the amplified human homologue

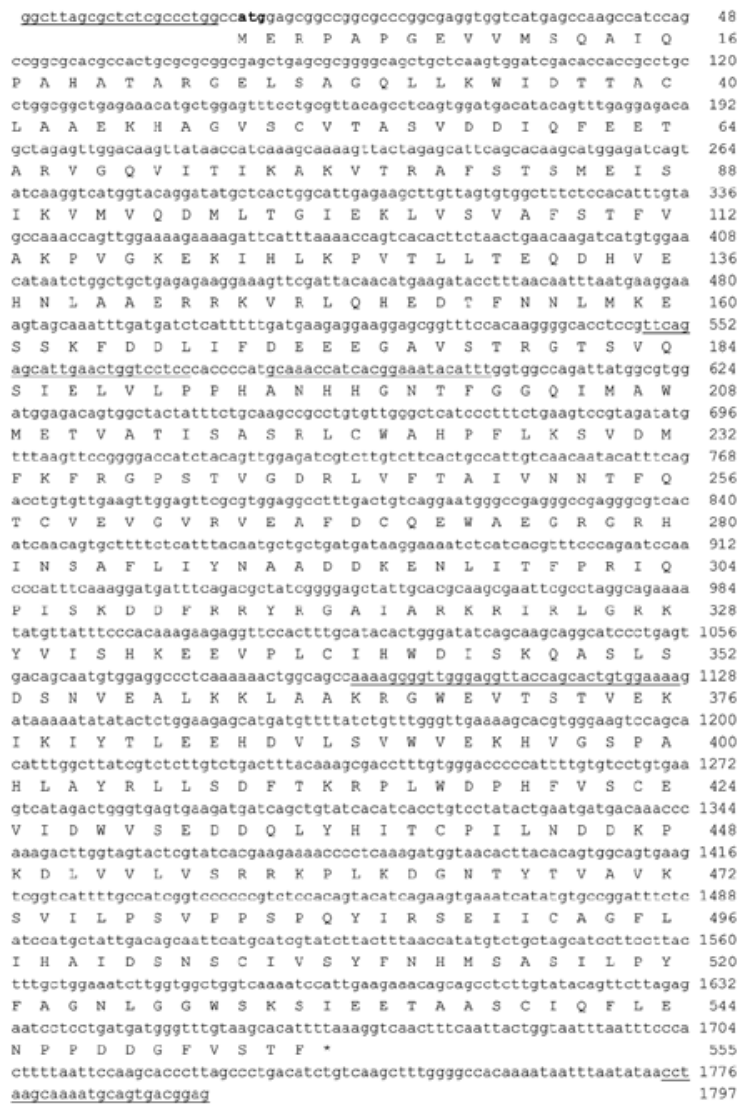

Figure 2. Nucleotide and deduced amino-acid sequences of the cDNA encoding full-length ORF of human cytosolic acetyl-CoA hydrolase.

The nucleotide and predicted amino-acid residues are numbered on the right from the first base of the ATG start codon (shown in bold face). The underlined nucleotide sequences correspond to the gene-specific primers used for sequencing (Table 1). The asterisk denotes the TAA stop codon. Sequence data have been submitted to DDBJ under the accession number AB078619. 


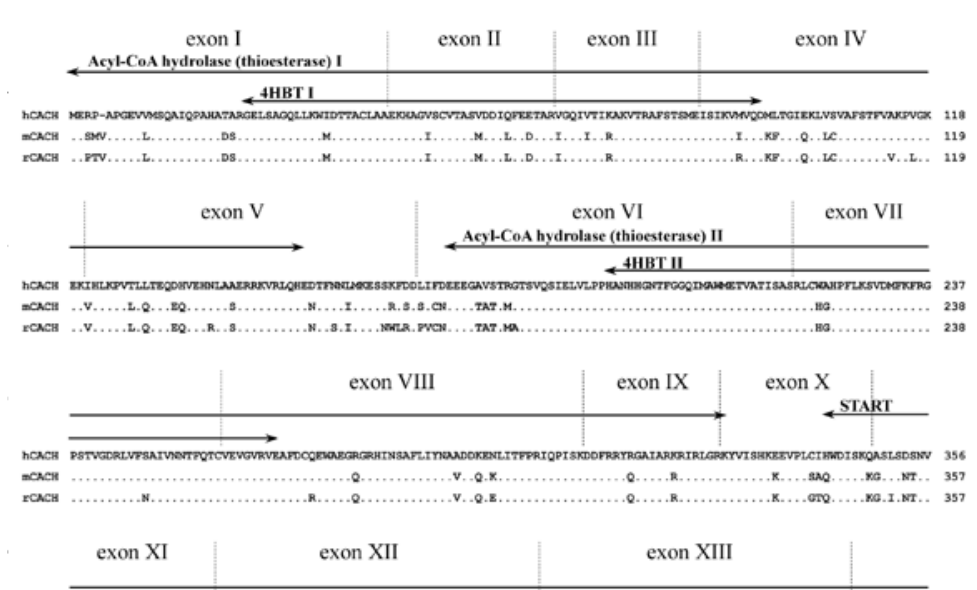

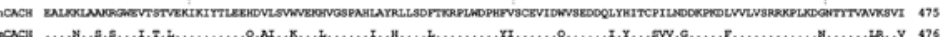

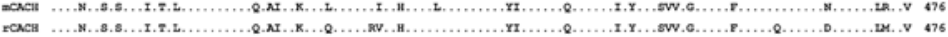

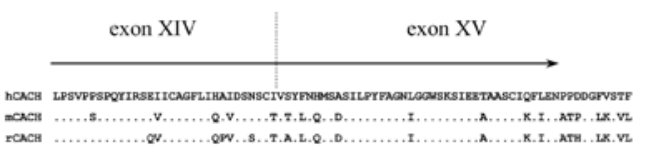

cDNA is $1820 \mathrm{bp}$ long and comprises the entire coding region of $1668 \mathrm{bp}$. The open reading frame (ORF) for a polypeptide of 555 amino-acid residues with a calculated molecular mass of $62033 \mathrm{Da}$, starts with an ATG initiation codon (Fig. 2, shown in bold face), whose first nucleotide is numbered as +1 , and terminates with a TAA stop codon (at positions 1669/1671, shown with an asterisk). The first AUG agrees pretty well with the Kozak's consensus sequence GCCGCC(A/G)CCAUGG for initiation in higher eukaryotes (Kozak, 1987; 1991). As shown in Fig. 3, the deduced amino-acid sequence of the human homologue cDNA exhibited extensive homology throughout the entire ORF to those of the mouse and rat $\mathrm{CACH}(81.4 \%$ and $78.7 \%$ identity, respectively, see Table 2). The human cDNA sequence is available from DDBJ/EMBL/GenBank under the accession number AB078619.

\section{Expression and purification of recombinant $\mathrm{CACH}$ in $S f 9$ insect cells}

Recombinant expression of the human $\mathrm{CACH}$ cDNA in Sfg insect cells resulted in overproduction of a His-tag fusion protein with the expected en-

Table 2. Comparison of nucleotide and deduced aminoacid sequence of cytosolic acetyl-CoA hydrolase ORF among human and the rodents.

Percentage identities of nucleotide and amino-acid residues are shown. The compared sequences are described in the legend to Fig. 3.

Length Identical residues with human $\mathrm{CACH}$

hCACH 555 aa

mCACH 556 aa $1371 / 1665$ bp (82.3\%) $452 / 555$ aa $(81.4 \%)$

rCACH 556 aa $1356 / 1665$ bp $(81.4 \%) \quad 437 / 555$ aa $(78.7 \%)$
Figure 3. Comparison of the deduced amino-acid sequence of cytosolic acetyl-CoA hydrolase among human and the rodent species.

The amino-acid residues are numbered on the right. Residues identical with those in the first sequence were denoted by dots. A dash has been introduced to maximize alignment. Exons I-XV are presented with their boundaries indicated by broken line. Arrows represent the extent of functional domains revealed by a publicly available database at the NCBI Conserved Domain Search: Acyl-CoA hydrolase (thioesterase); 4HBT (4hydroxybenzoyl-CoA thioesterase); START (steroidgenic acute regulatory (StAR)-related lipid-transfer) domain. The compared sequences are as follows: hCACH, human cytosolic acetyl-CoA hydrolase (GenBank accession $\mathrm{AB} 078619) ; \mathrm{mCACH}$, mouse cytosolic acetyl-CoA hydrolase (GenBank accession $\mathrm{AB} 078618)$; $\mathrm{rCACH}$, rat cytosolic sss acetyl-CoA hydrolase (GenBank accession ${ }_{556}^{55}$ AB040609).

zymatic activity. The activity was detected as early as two days after infection in the lysate prepared from infected cells, and was maximal at four days post-infection. The expressed human recombinant enzyme hydrolyzed acetyl-CoA in the presence of $2 \mathrm{mM}$ ATP and the activity was completely inhibited by ADP. Further, it exhibited cold lability and its cold-inactivation could be partially abolished through the incubation at $37^{\circ} \mathrm{C}$ in the presence of $0.16 \mathrm{mM}$ Triton X-100 just as the rodent enzymes (Suematsu et al., 2001; 2002). The expressed His-tag

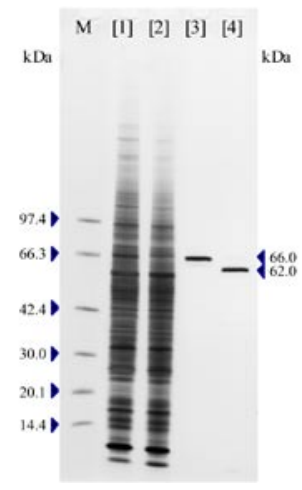

Figure 4. SDS/PAGE analysis of purified recombinant hCACH protein.

Protein samples from purification steps for the recombinant hCACH protein were subjected to SDS/PAGE on a $10-20 \%$ polyacrylamide gradient gel followed by silver staining. The samples, prepared as described in Materials and Methods, were the clarified cell lysate from the infected insect cells [1], the flow through from the $\mathrm{Ni}^{2+}$-charged resin [2], the eluted His-tag fusion protein [3] and the $\mathrm{hCACH}$ protein free of the fused peptide [4]. Numbers to the left and right denote the molecular mass (in $\mathrm{kDa}$ ) of the markers (lane M) and the purified proteins (lanes [3] and [4]). The molecular mass markers are phosphorylase $b$ (97400), bovine serum albumin (66300), aldolase (42400), carbonic anhydrase (30300), soybean trypsin inhibitor (20100) and lysozyme (14400). 
Table 3. Affinity purification and characterization of a recombinant form of human cytosolic acetyl-CoA hydrolase.

(A) Recombinant human cytosolic acetyl-CoA hydrolase protein expressed in infected $S f 9$ insect cells $(4 \mu \mathrm{l}$ cell pellet) was purified as described in the text. (B) Constants representing catalytic activity of purified human recombinant enzyme $(\mathrm{hCACH})$ and purified rat counterpart $(\mathrm{rCACH}$, Suematsu et al., 2001). Proteins were determined according to the method of Bradford.

(A)

\begin{tabular}{llllll}
\hline Step & $\begin{array}{l}\text { Total } \\
\text { activity } \\
(\mathrm{U})\end{array}$ & $\begin{array}{l}\text { Total pro- } \\
\text { tein } \\
(\mu \mathrm{g})\end{array}$ & $\begin{array}{l}\text { Specific } \\
\text { activity } \\
\left(\mathrm{U} \cdot \mathrm{mg}^{-1}\right)\end{array}$ & $\begin{array}{l}\text { Purifi- } \\
\text { cation } \\
(- \text {-fold })\end{array}$ & $\begin{array}{l}\text { Yield } \\
(\%)\end{array}$ \\
\hline Lysate & 5.2 & 655 & 7.9 & - & 100 \\
Eluate & 5.0 & 1.51 & 3300 & 419 & 97 \\
\hline
\end{tabular}

(B)

\begin{tabular}{lllll}
\hline Enzyme & $\begin{array}{l}\text { Specific } \\
\text { activity } \\
\left(\mathrm{U} \cdot \mathrm{mg}^{-1}\right)\end{array}$ & $\begin{array}{l}\text { Michaelis } \\
\text { constant }\end{array}$ & $\begin{array}{l}\text { Catalytic } \\
K_{\mathrm{m}}(\mathrm{M})\end{array}$ & $\begin{array}{l}\text { Constant } \\
k_{\text {cat }}\left(\mathrm{s}^{-1}\right)\end{array}$ \\
$k_{\text {cat }} / K_{\mathrm{m}}\left(\mathrm{M}^{-1} \cdot \mathrm{s}^{-1}\right)$ \\
\hline $\mathrm{hCACH}$ & 3300 & $1.5 \times 10^{-4}$ & $7.0 \times 10^{3}$ & $4.6 \times 10^{7}$ \\
$\mathrm{rCACH}$ & 3100 & $1.5 \times 10^{-4}$ & $6.6 \times 10^{3}$ & $4.3 \times 10^{7}$ \\
\hline
\end{tabular}

fusion protein was purified to homogeneity with $97 \%$ yield by affinity purification using $\mathrm{Ni}^{2+}$-charged resin (Table 3A) and then relieved of the fused peptide by thrombin cleavage (Fig. 4). The purified human $\mathrm{CACH}$ represented comparable specific activity $(3300 \mathrm{U} / \mathrm{mg})$, Michaelis constant for acetyl-CoA $\left(K_{\mathrm{m}}\right.$ $\left.=1.5 \times 10^{-4} \mathrm{M}\right)$, catalytic constant $\left(k_{\text {cat }}=7.0 \times 10^{3} / \mathrm{s}\right)$ and catalytic efficiency $\left(k_{\text {cat }} / K_{\mathrm{m}}=4.6 \times 10^{7} / \mathrm{M} \cdot \mathrm{s}\right)$ to those of the rat enzyme (Table 3B).

Highly conserved genomic organization of mammalian $\mathrm{CACHs}$

We here describe a comparative analysis of the genomic organization of human, mouse and rat CACHs. It was verified by mRNA-genomic alignments and published in established databases that the $\mathrm{CACH}$ gene is mapped at human chromosome 5 q14.1, mouse chromosome $13 \mathrm{C} 3$ and rat chromosome $2 \mathrm{q} 12$ and covers about $64-\mathrm{kb}, 45-\mathrm{kb}$ and $50-\mathrm{kb}$ genomic regions, respectively (Fig. 5). The mammalian $\mathrm{CACH}$ genes are similarly composed of 15 exons, the size of each of which is identical among the three mammalian species with an exception that exon I codes for 43 amino-acid residues in human and 44 residues in the rodents (Fig. 3). Introns are generally longer in human than in the rodents with the exceptions of introns I, IX, and X (Fig. 5).

\section{Putative domain structure of mammalian $\mathrm{CACH}$ proteins}

Bioinformatical work using the NCBI Conserved Domain Search website revealed that human, mouse and rat $\mathrm{CACH}$ proteins commonly consist of uniquely aligned functional domains as follows: two acyl-CoA hydrolase (thioesterase) regions (accession: COG1607 (Dillon \& Bateman, 2004)) encoded by exons I-V and VI-IX, respectively, and a C-terminal Steroidgenic acute regulatory (StAR) related lipid transfer (START) domain (accession: pfam01852; (Tsujishita \& Hurley, 2000)) encoded by exons XXV (Fig. 3). Because the rest of the domains might be too small, ranging in length from only 10 to 23 amino-acid residues, to contribute to the enzymatic function, all or some of the three functional domains presumably contribute to the $\mathrm{CACH}$ activity. As shown in Fig. 3, 4-hydroxybenzoyl-CoA thioesterase (4HBT) domains (accession: pfam03061) I, II, encoded by exons I-IV and VI-VIII, respectively, were also demarcated in the midst of the thioesterase regions I and II, respectively. The 4HBT domains I and II show especially high amino-acid sequence homology between human and mouse/rat (I: $87.5 \% / 86.1 \%$ identity; II: $93.2 \% / 93.2 \%$ identity). On the other hand, the C-terminal START domain shows a relatively low amino-acid sequence homology between human and mouse/rat (77.1\%/74.6\% identity).

\section{Characterization of the putative promoter region of mammalian $\mathrm{CACH}$ genes}

The putative promoter regions of human, mouse and rodent $\mathrm{CACH}$ genes are unexpectedly found to be of the housekeeping-type, namely GCrich with GC box(es), but lacking both TATA and CCAAT boxes, as described below. The 5'-flanking nucleotide sequences $100 \mathrm{bp}$ long show high GC contents (human, $83 \%$; mouse, $74 \%$; rat, $70 \%$ ). A GC box (GGGCGG) is commonly found in the upstream regions of the three mammalian species, especially in human, where six tandemly arranged GC boxes are found in the region $-107 /-54$ (Fig. 6). On the other hand, although we searched the $5^{\prime}$ -

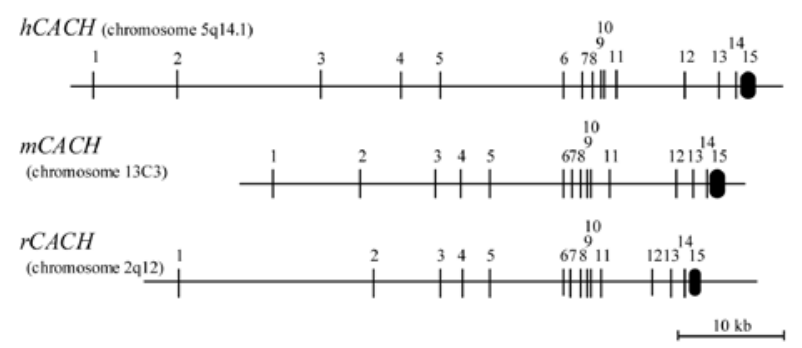

Figure 5. Comparison of exon-intron structure of cytosolic acetyl-CoA hydrolase genes among human and the rodents.

Exons are numbered from 1 to 15 . The data here taken from publicly available RefSeq genes as follows: $h C A C H$, human cytosolic acetyl-CoA hydrolase (GenBank accession NM_130767); $m C A C H$, mouse cytosolic acetyl-CoA hydrolase (GenBank accession NM_028790); rCACH, rat cytosolic acetyl-CoA hydrolase (GenBank accession NM_130747). 


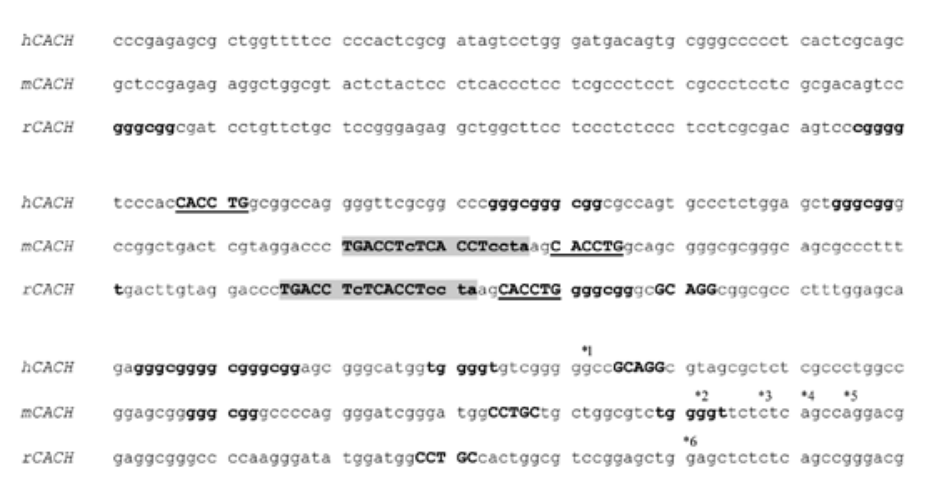

$-141$

$-141$

$-141$

$-71$

$-71$

$-71$

$-1$

ized cis-elements are commonly found in the upstream regions of the mammalian $\mathrm{CACH}$ genes (Fig. 6). These features observed are discussed later in relation to the $\mathrm{CACH}$ expression.

\section{DISCUSSION}

A human cytosolic acetyl-CoA hydrolase $(\mathrm{CACH}) \mathrm{cDNA}$ was cloned from a liver cDNA library and characterized

Figure 6. Comparison of the 5'-upstream region of cytosolic acetyl-CoA for the first time. Cytoplasmic localizahydrolase genes among human and the rodents.

The nucleotide residues are numbered on the right, relative to the adenine residue of the AUG translational start triplet marked as +1 . Asterisks denote initiation sites of transcripts (*3, AK034622; *4, AK004905, AK033663; *6, AB040609) or ESTs *1, AV693695, AV693776; *2, BB843576; *4, BB626692, BY705110; *5, BB625271) from GenBank. Consensus binding sites are indicated with bold letters: 'GC boxes' gggcgg for Sp1; 'E box' CACCTG for bHLH is underlined; cggggt/tggggt for Adr1p; CCTGC for LVc (leukemia virus factor c); PPRE TGACCTcTCACCTccta is shaded. The compared sequences are described in the legend to Fig. 5.

flanking region of the $\mathrm{CACH}$ genes up to the position -1200 , neither a canonical core promoter motif TATA box (TATAAA) nor a CCAAT box was found in mouse and rat. These cis-elements were also absent in the proximal promoter region of the human gene, whereas in a farther upstream region, a sequence TTTATA at $-824 /-819$, corresponding to the inverted sequence of TATAAA, a typical CCAAT box at $-394 /-390$, and its inverted sequence ATTGG at $-767 /-763$, were found.

A peroxisome proliferator responsive element (PPRE) motif (TGACCTcTGACCT) (Kliewer et al., 1992) was, as expected, found in the rodent $\mathrm{CACH}$ promoter regions (Fig. 6), consistent with the marked $\mathrm{CACH}$ induction by peroxisome proliferator (PP) observed in rat liver (Prass et al., 1980). In human the PPRE was found not in the putative promoter region but in introns II and III and so forth of the $\mathrm{CACH}$ gene (Table 4). Further, a computer analysis using TESS revealed that possible well-character- tion of the human $\mathrm{CACH}$ was predicted by PSORT WWW Server from the cDNA nucleotide sequence not containing any targeting signals for organella including peroxisome, mitochondria or nucleus, as well as the rodent enzymes (Suematsu et al., 2001; 2002). The extensive homology of its deduced amino-acid sequence with the rodent enzymes (Table 2) implies that the obtained human homologue cDNA encodes no other acyl-CoA thioesterase (Broustas et al., 1996; Hunt et al., 1999) but $\mathrm{CACH}$. The authenticity of the human cDNA was further confirmed by its functional expression. The expressed recombinant form of human $\mathrm{CACH}$ was found to be a cold labile allosteric enzyme activated by ATP and completely inhibited by ADP, and shared comparable enzymatic characteristics with the rat enzyme (Table 3B). Thus, our previous and current studies clearly demonstrate that the mammalian $\mathrm{CACH}$ proteins have been highly conserved.

Here we proposed putative functional domains of $\mathrm{CACH}$ (Fig. 3). Among them, the highly conserved 4HBT thioesterase domains (Benning et al., 1998) presumably contribute greatly to the enzymatic activity of active $\mathrm{CACH}$ homooligomers. Although the actual domain functions remain to be established, the highly conserved unique alignment of the putative functional domains (Fig. 3) together with the overall high amino-acid sequence similar-

Table 4. Putative peroxisome proliferator responsive elements (PPRE) in human, mouse and rat cytosolic acetyl-CoA hydrolase genes.

The nucleotide sequences are described in the legend to Fig. 5. PPRE was searched using TESS. Nucleotide positions are numbered as in Fig. 2. Boldface letters represent highly conserved sequences in agreement with the consensus sequences for PPRE (Kliewer et al., 1992).

\begin{tabular}{llll}
\hline Gene & Sequence $\left(5^{\prime}-3^{\prime}\right)$ & Position in gene & Location \\
\hline \multirow{2}{*}{$h C A C H$} & ggtaAGGTCAtAGATCA & $-39,165 /-39,149$ & $5^{\prime}$-upstream region \\
& aggtTTGTCAaAGGTCA & $15,930 / 15,946$ & intron II \\
& agtcAGGTCAcAGGTAA & $22,527 / 22,543$ & intron III \\
& TGACCAtTGTCCTgcat & $-266 /-249$ & $5^{\prime}$-flanking region \\
& TGACCTCTCACCTccta & $-101 /-85$ & $5^{\prime}$-flanking region \\
& TGACCTcTCACCTcta & $-105 /-89$ & $5^{\prime}$-flanking region \\
& attcAGGTCAaAGTTCA & $33,614 / 33,630$ & intron V \\
\hline
\end{tabular}


ity among the three mammalian species (Table 2) implies a crucial role of $\mathrm{CACH}$ in mammalian metabolism.

In the mammalian $\mathrm{CACH}$ genes, all the introns are found to be bordered by consensus GTAG splice sites, which are usually excised by the major spliceosome utilizing U2 snRNA (Sharp \& Burge, 1997), and further their splicing donor and acceptor sites share common pyrimidine stretches (Breathnach et al., 1978; Breathnach \& Chambon, 1981). In the human CACH gene, as shown in Table 5 , exons range in length from $56 \mathrm{bp}$ (exon IX) to 157 bp (exon VI), while the introns from $270 \mathrm{bp}$ (intron XIV) to $13946 \mathrm{~kb}$ (intron II). As commonly found in the mammalian $\mathrm{CACH}$ genes, introns $\mathrm{I}-\mathrm{V}$ are generally larger, whereas introns VI-X are distinctly compact (Fig. 5), implying separate origins of the two thioesterase domains, composed of exons $\mathrm{I}-\mathrm{V}$ and VI-IX, respectively, and their conjugation by domain shuffling during evolution of the genomes. This interesting inference is further supported by the finding that intron $\mathrm{V}$ located at the domain border is of phase I, which is classified according to the number of bases of the final codon generated in the previous exon (Cawley et al., 2001). It has been proposed that domain shuffling within phase I introns played an important role in the evolution of the human genome (Kaessmann et al., 2002).

The three major core promoter motifs containing binding sites for RNA polymerase II have been reported to be the TATA box, the initiator and the downstream promoter element (Burke \& Kadonaga, 1997). In the present paper, we analyzed mammalian $\mathrm{CACH}$ putative promoter regions for the first time. To estimate the transcription start sites of $\mathrm{CACH}$, each 5'-end of mammalian $\mathrm{CACH}$ transcripts and EST entries extending into the $5^{\prime}$-direction available from the GenBank was marked on the $5^{\prime}$-upstream region of the mammalian $\mathrm{CACH}$ genes (Fig. 6). Unexpectedly, we found that none of them matched the consensus sequence of mammalian initiator PyPy- $A_{+1}-N-T / A-P y-P y$, whose $A_{+1}$ is the transcription start site (Burke \& Kadonaga, 1997). We here report that the $\mathrm{CACH}$ putative promoters do not have any of the three known core promoter motifs, suggesting the presence of other core promoter motifs responsible for accurate positioning of RNA polymerase relative to the initiation site.

It should be noted that a number of promoters lack classical TATA or CCAAT boxes but have an increased GC content with specificity protein 1 (Sp 1) binding site(s), features typical of 'housekeeping' genes (La Thangue \& Rigby, 1988; Rundlöf et al., 2001). The ubiquitously expressed TATA-less genes have been reported to be controlled by $\mathrm{Sp}$ proteins (Dynan et al., 1986; Suske, 1999; Müller et al., 1999), which have been shown to bind the GC box, the hexanucleotide sequence GGGCGG (Lania et al., 1997). Interestingly, the mammalian $\mathrm{CACHs}$ have a typical housekeeping-type promoter (Fig. 6), which presumably stimulates constitutive transcription of $\mathrm{CACH}$, implying that the enzyme might be generally essential for mammalian cells. The ubiquitously expressed Sp1 was reported as a member of a transcriptionally active multiprotein-DNA complex (Foti et al., 2003), and also as a repressor by recruiting histone deacetylase 1 (HDAC1) to the growth-regulated murine thymidine kinase gene (TK) promoter (Doetzlhofer et al., 1999). Thus, it is likely that Sp1 acts in a combinatorial manner with other transcription factors, which may have more pronounced temporally or spatially restricted expression patterns. It has been also shown that the Sp1-related BTEB (basic transcription element binding protein) protein is a repressor of a promoter containing a single GC box, however, when the GC box was repeated five times, BTEB turned out to be an activator (Lania et al., 1997). Thus, Sp1-related proteins like BTEB could possibly influence the $\mathrm{CACH}$ transcription differently between the rodents and human, since the former contains a single GC box and the latter contains six tandemly arranged GC boxes in their putative promoter regions of $\mathrm{CACH}$. How the Sp proteins actually control the $\mathrm{CACH}$ gene expression remains to be established.

Although the mammalian CACHs have a typical housekeeping-type promoter, the enzyme activity is detectable only in liver and kidney of the rat tissues tested (Matsunaga et al., 1985). The tissuespecific expression must arise from cis-acting elements other than a GC box. Web-based search using TESS revealed several other possible cis-elements commonly found in the mammalian $\mathrm{CACH}$ putative promoter regions (Fig. 6) as described below. First, consensus (C/T)GGGGT sequence, known as a binding site of yeast Adr1p, locates at $-42 /-37$ (human), $-22 /-17$ (mouse) and -145/-140 (rat). Adr1p has been reported to govern fatty acid degradation and peroxisome proliferation (Gurvitz et al., 2001). Second, consensus CCTGC sequence, known as a binding site of LVc (leukemia virus factor c), lies at $-26 /-22$ (human), -37/-33 (mouse) and -43/-39, -92/-88 (rat). LVc interacts with leukemia virus enhancer (Speck \& Baltimore, 1987). Third, an E box motif (consensus CANNTG) exists at $-134 /-129$ (human), $-101 /-96$ (mouse) and -106/-101 (rat). It is usually found in promoter or enhancer regions and is known as a binding site of ubiquitous basic helix-loop-helix (bHLH) transcription factors, which induce the expression of a number of genes in the appropriate cell type, resulting in a tissue-specific phenotype (Murre et al., 1994). The bHLH proteins can be categorized into three classes: Class A and B function as transcriptional activators, while Class $\mathrm{C}$ as transcrip- 
tional repressors (Azmi et al., 2003). Since E box sites appear in a wide variety of promoters and enhancer regions (Murre et al., 1994), other flanking factors would specify their functions. In the rodent $\mathrm{CACH}$ promoter region, an E box is located close to a PPRE element, and in rat it is additionally found directly adjacent to a GC box (Fig. 6), suggesting possible synergistic transactivation of the $\mathrm{CACH}$ gene by their ligands. A PPRE element found in the rodent $\mathrm{CACHs}$ putative promoter region (Fig. 6) must be responsible for the marked enzyme induction by a peroxisome proliferator (PP) (Prass et al., 1980). On the other hand, the human PPRE elements found outside of the putative promoter region (Table 4) remain to be determined whether active or not. A significant number of studies have suggested that human hepatocytes are non-responsive to PPs - this includes epidemiological studies using hypolipidemic drugs, and in vitro experiments with human hepatocytes (Tugwood et al., 1996). To address the question whether hCACH is induced by PPs, further in vitro experiments with human hepatocytes should be carried out. Precise demarcation of the promoter and identification of all the elements involved in the gene transcription awaits further experiments including in vivo transfection.

In conclusion, this paper for the first time describes the entire cDNA sequence of human cytosolic acetyl-CoA hydrolase and its overproduction, allowing future studies on its physiological functions and physicochemical characteristics. Although a detailed characterization of its promoter remains to be established, the bioinformatical promoter analysis presented here has provided insights into the regulation of the gene expression. Transgenic approach is currently underway to explore physiological roles of the enzyme. The in vivo study would provide direct evidence on the enzyme functions and facilitate conclusive establishment of its significance.

\section{Acknowledgements}

This research was supported by a Grant-inAid for Scientific Research from the Ministry of Education, Science, Sports and Culture of Japan and by a grant from the Vitamin Society of Japan.

\section{REFERENCES}

Azmi S, Sun H, Ozog A, Taneja R (2003) mSharp-1/DEC2, a basic helix-loop-helix protein functions as a transcriptional repressor of E box activity and Stra13 expression. J Biol Chem 278: 20098-20109.

Benning MM, Wesenberg G, Liu R, Taylor KL, DunawayMariano D, Holden HM (1998) The three-dimensional structure of 4-hydroxybenzoyl-CoA thioesterase from Pseudomonas sp. strain CBS-3. J Biol Chem 273: 3357233579.
Berge RK, Aarsland A, Bakke OM, Farstad M (1983) Hepatic enzymes, CoASH and long-chain acyl-CoA in subcellular fractions as affected by drugs inducing peroxisomes and smooth endoplasmic reticulum. Int J Biochem 15: 191-204.

Breathnach R, Chambon P (1981) Organization and expression of eucaryotic split genes coding for proteins. Annu Rev Biochem 50: 349-383.

Breathnach R, Benoist C, O'Hare K, Gannon F, Chambon P (1978) Ovalbumin gene: evidence for a leader sequence in mRNA and DNA sequences at the exon-intron boundaries. Proc Natl Acad Sci USA 75: 4853-4857.

Broustas CG, Larkins LK, Uhler MD, Hajra AK (1996) Molecular cloning and expression of cDNA encoding rat brain cytosolic acyl-coenzyme A thioester hydrolase. J Biol Chem 271: 10470-10476.

Burke TW, Kadonaga JT (1997) The downstream core promoter element, DPE, is conserved from Drosophila to humans and is recognized by $\mathrm{TAF}_{\mathrm{II}} 60$ of Drosophila. Genes Dev 11: 3020-3031.

Cawley SE, Wirth AI, Speed TP (2001) Phat-a gene finding program for Plasmodium falciparum. Mol Biochem Parasitol 118: 167-174.

Dillon SC, Bateman A (2004) The Hotdog fold: wrapping up a superfamily of thioesterases and dehydratases. BMC Bioinformatics 5: 109-122.

Doetzlhofer A, Rotheneder H, Lagger G, Koranda M, Kurtev V, Brosch G, Wintersberger E, Seiser C (1999) Histone deacetylase 1 can repress transcription by binding to Sp1. Mol Cell Biol 19: 5504-5511.

Dynan WS, Sazer S, Tjian R, Schimke RT (1986) Transcription factor $S p 1$ recognizes a DNA sequence in the mouse dihydrofolate reductase promoter. Nature 319: 246-248.

Ebisuno S, Isohashi F, Nakanishi Y, Sakamoto Y (1988) Acetyl-CoA hydrolase: relation between activity and cholesterol metabolism in rat. Am J Physiol 255: R724R730.

Ebisuno S, Isohashi F, Nakanishi Y, Higashi T, Sakamoto Y (1989) The biphasic change of cytosolic acetyl-CoA hydrolase in rat liver during 3 '-methyl-4-dimethylaminoazobenzene hepatocarcinogenesis. Jpn J Cancer Res 80: 132-135.

Foti D, Iuliano R, Chiefari E, Brunetti A (2003) A nucleoprotein complex containing Sp1, C/EBP $\beta$, and HMGI$\mathrm{Y}$ controls human insulin receptor gene transcription. Mol Cell Biol 23: 2720-2732.

Gurvitz A, Hiltunen JK, Erdmann R, Hamilton B, Hartig A, Ruis H, Rottensteiner H (2001) Saccharomyces cerevisiae Adr1p governs fatty acid $\beta$-oxidation and peroxisome proliferation by regulating POX1 and PEX11. J Biol Chem 276: 31825-31830.

Horie S, Isobe M, Suga T (1986) Changes in CoA pools in hepatic peroxisomes of the rat under various conditions. J Biochem 99: 1345-1352.

Hunt MC, Nousiainen SEB, Huttunen MK, Orii KE, Svensson LT, Alexson SEH (1999) Peroxisome proliferatorinduced long chain acyl-CoA thioesterases comprise a highly conserved novel multi-gene family involved in lipid metabolism. J Biol Chem 274: 34317-34326.

Isohashi F, Nakanishi Y, Sakamoto Y (1983a) Factors affecting the cold inactivation of an acetyl-Coenzyme-A hydrolase purified from the supernatant fraction of rat liver. Eur J Biochem 134: 447-452.

Isohashi F, Nakanishi Y, Sakamoto Y (1983b) Effects of nucleotides on a cold labile acetyl-CoA hydrolase from the supernatant fraction of rat liver. Biochemistry 22: 584-590. 
Isohashi F, Nakanishi Y, Matsunaga T, Sakamoto Y (1984) A cold-labile acetyl-coenzyme-A hydrolase from the supernatant fraction of rat liver: reactivation and reconstitution of the active species from the inactive monomer. Eur J Biochem 142: 177-181.

Kaessmann H, Zöllner S, Nekrutenko A, Li W-H (2002) Signatures of domain shuffling in the human genome. Genome Res 12: 1642-1650.

Kliewer SA, Umesono K, Heyman RA, Mangelsdorf DJ, Dyck JA, Evans RM (1992) Retinoid X receptor-COUPTF interactions modulate retinoic acid signaling. Proc Natl Acad Sci USA 89: 1448-1452.

Kozak M (1987) At least six nucleotides preceding the AUG initiator codon enhance translation in mammalian cells. J Mol Biol 196: 947-950.

Kozak M (1991) Structural features in eukaryotic mRNAs that modulate the initiation of translation. J Biol Chem 266: 19867-19870.

La Thangue NB, Rigby PWJ (1988) Trans-acting protein factors and the regulation of eucaryotic transcription. In Transcription and Splicing (Hames BD, Glover DM, eds) pp 1-42, IRL Press, Washington, DC.

Lania L, Majello B, De Luca P (1997) Transcriptional regulation by the Sp family proteins. Int J Biochem Cell Biol 29: 1313-1323.

Mannaerts GP, Debeer LJ, Thomas J, De Schepper PJ (1979) Mitochondrial and peroxisomal fatty acid oxidation in liver homogenates and isolated hepatocytes from control and clofibrate-treated rats. J Biol Chem 254: 45854595.

Matsunaga T, Isohashi F, Nakanishi Y, Sakamoto Y (1985) Physiological changes in the activities of extramitochondrial acetyl-CoA hydrolase in the liver of rats under various metabolic conditions. Eur J Biochem 152: 331-336.

Müller C, Yang R, Beck-von-Peccoz L, Idos G, Verbeek W, Koeffler HP (1999) Cloning of the cyclin A1 genomic structure and characterization of the promoter region. J Biol Chem 274: 11220-11228.

Murre C, Bain G, van Dijk MA, Engel I, Furnari BA, Massari ME, Matthews JR, Quong MW, Rivera RR, Stuiver $\mathrm{MH}$ (1994) Structure and function of helix-loop-helix proteins. Biochim Biophys Acta 1218: 129-135.

Nakanishi Y, Isohashi F, Ebisuno S, Sakamoto Y (1988) Binding of nucleotides to an extramitochondrial acetylCoA hydrolase from rat liver. Biochemistry 27: 48224826.

Nakanishi Y, Okamoto K, Isohashi F (1993) Effects of chronic administration of the peroxisome proliferator, clofibrate, on cytosolic acetyl-CoA hydrolase in rat liver. Biochem Pharmacol 45: 1403-1407.

Nakanishi Y, Okamoto K, Isohashi F (1994) Subcellular distribution of ATP-stimulated and ADP-inhibited
acetyl-CoA hydrolase in livers from control and clofibrate-treated rats: comparison of the cytosolic and peroxisomal enzyme. J Biochem 115: 328-332.

Prass RL, Isohashi F, Utter MF (1980) Purification and characterization of an extramitochondrial acetyl coenzyme A hydrolase from rat liver. J Biol Chem 255: 5215-5223.

Rundlöf A-K, Carlsten M, Arnér ESJ (2001) The core promoter of human thioredoxin reductase 1. J Biol Chem 276: 30542-30551.

Schug J, Overton GC (1997) TESS: Transcription Element Search Software on the WWW. Website (http://www.cbil. upenn.edu/tess/) Computational Biology and Informatics Laboratory School of Medicine University of Pennsylvania. Technical Report CBIL-TR-1997-1001-v0.0.

Sharp PA, Burge CB (1997) Classification of introns: U2type or U12-type. Cell 91: 875-879.

Simon EJ, Shemin D (1953) The preparation of S-succinyl Coenzyme A. J Am Chem Soc 75: 2520.

Söling H-D, Rescher C (1985) On the regulation of cold-labile cytosolic and of mitochondrial acetyl-CoA hydrolase in rat liver. Eur J Biochem 147: 111-117.

Speck NA, Baltimore D (1987) Six distinct nuclear factors interact with the 75-base-pair repeat of the Moloney murine leukemia virus enhancer. Mol Cell Biol 7: 11011110.

Suematsu N, Okamoto K, Isohashi F (1996) Effects of various proteins or peptides on reactivation of cold-inactivated acetyl-CoA hydrolase from rat liver. St Marianna Med J 24: 691-697.

Suematsu N, Okamoto K, Shibata K, Nakanishi Y, Isohashi F (2001) Molecular cloning and functional expression of rat liver cytosolic acetyl-CoA hydrolase. Eur J Biochem 268: 2700-2709.

Suematsu N, Okamoto K, Isohashi F (2002) Mouse cytosolic acetyl-CoA hydrolase, a novel candidate for a key enzyme involved in fat metabolism: cDNA cloning, sequencing and functional expression. Acta Biochim Polon 49: 937-945.

Suematsu N, Okamoto K, Isohashi F (2003) Simple and unique purification by size-exclusion chromatography for an oligomeric enzyme, rat liver cytosolic acetylcoenzyme A hydrolase. J Chromatogr B, Analyt Technol Biomed Life Sci 790: 239-244.

Suske G (1999) The Sp-family of transcription factors. Gene 238: 291-300.

Tsujishita Y, Hurley JH (2000) Structure and lipid transport mechanism of a StAR-related domain. Nat Struct Biol 7: 408-414.

Tugwood JD, Aldridge TC, Lambe KG, Macdonald N, Woodyatt NJ (1996) Peroxisome proliferator-activated receptors: structures and function. Ann N Y Acad Sci 804: 252-265. 